\title{
Fatal Bleeding in Conjunction with Mandibular Medication-related Osteonecrosis of the Jaw (MRONJ)
}

\author{
Taiki Suzuki $^{1,2)}$, Ryo Sekiya ${ }^{1)}$, Yuji Hamada ${ }^{1)}$, Miho Takahashi ${ }^{1)}$, \\ Kazunari Karakida ${ }^{1)}$ and Haruo Sakamoto ${ }^{1)}$ \\ ${ }^{1)}$ Department of Oral and Maxillofacial Surgery, Hachioji Hospital Tokai University, \\ 1838 Ishikawamachi, Hachioji, Tokyo 192-0032, Japan \\ 2) Department of Oral Medicine, Oral and Maxillofacial Surgery, Tokyo Dental College, \\ 5-11-13 Sugano, Ichikawa, Chiba 272-8513, Japan
}

Received 13 December, 2016/Accepted for publication 22 February, 2017

\begin{abstract}
Here, we report a case of fatal bleeding in conjunction with mandibular medicationrelated osteonecrosis of the jaw (MRONJ). A 75-year-old Japanese man was referred to our department with osteonecrosis of the jaw due to bisphosphonate (BP) for multiple bone metastases from prostate cancer. Aggressive surgical intervention was ruled out due to a poor prognosis in terms of life expectancy. Death occurred due to hemorrhagic shock resulting from massive oral bleeding caused by necrosis of the mandible. Numerous reports have suggested that jaw necrosis is induced not only by BP, but also RANKL antibody, steroids, and molecularly-targeted agents. This suggests that the number of cases of MRONJ is likely to increase among elderly patients in whom general health is already poor. The American Association of Oral and Maxillofacial Surgery recommends aggressive treatment only in cases of stage 3 disease. Therefore, such a therapeutic strategy may only be available for cases of jaw necrosis in which the general health status of the patient is otherwise good. To prevent a life-threatening outcome in cases of MRONJ, physicians, who are responsible for determining the drug strategy, should cooperate with oral surgeons in determining the best therapeutic strategy.
\end{abstract}

Key words: Medication-related osteonecrosis of the jaw -

Bisphosphonate (BP)-related osteonecrosis of the jaw-

Anti-cancer treatment-Complications

\section{Introduction}

The role of bisphosphonate (BP) in the development of osteonecrosis of the jaw $(\mathrm{ONJ})$ is now well known among doctors, who are responsible for prescribing this type of medication, dentists, oral surgeons, and even patients. This is due to the publication of a position paper on BP-related ONJ (BRONJ) ${ }^{32)}$.

Recent studies have suggested that ONJ is induced not only by oral BP, but also by RANKL monoclonal antibody drugs ${ }^{11,23)}$, ste- 
roids, non-oral BP drugs, and molecularlytargeted agents $^{14,15,18,22)}$.

Hospitals are now seeing an increase in the number of cases if non-BP related ONJ. In 2014, the American Association of Oral and Maxillofacial Surgeons (AAOMS) acknowledged the scope of this change in our understanding of the etiology of this condition by changing its name to medication-related osteonecrosis of the jaw (MRONJ) ${ }^{25)}$. In the AAOMS position paper ${ }^{25)}$, symptomatic treatment, mouth rinse, and pain control are recommended as the main methods of management up until stage 2 disease; scraping is limited to only soft tissue; and aggressive surgical procedures are not recommended, except in stage- 3 patients. We believe that adherence to this strategy is likely to result in an increase in the number of cases of uncontrolled ONJ.

Here, we report an elderly patient who died of hemorrhagic shock due to massive oral bleeding as a result of lower jaw necrosis. At the time, the patient was on BP for treatment of bone metastasis from prostate cancer. The patient, who was followed up over a long period of time, received no aggressive surgical intervention due to a poor prognosis for life expectancy.

Further investigation of the diagnosis and a new therapeutic strategy will be discussed.

\section{Case Presentation}

Patient: A 75-year-old man.

Medical history: The patient had had heart failure and prostate cancer, and had received hormone therapy and zoledronic acid for approximately 1 year after being diagnosed with multiple bone metastases. The cancer was unresponsive to hormone therapy, however. Therefore, concurrent hormone therapy and docetaxel administration were selected, which is the standard treatment in castrationresistant cases (Fig. 1, Table 1 ).

Chief complaint: Pain in the tooth due to an apical lesion in the mandibular left molar.

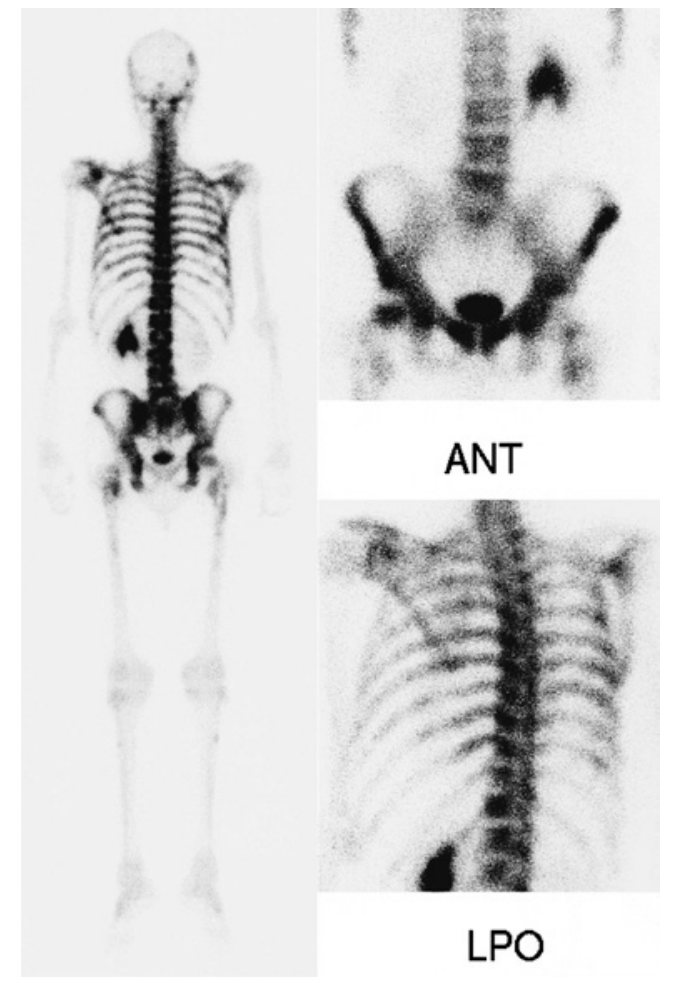

Fig. 1 Bone scintigraphy

Accumulation of R1 in cervical vertebrae, spinal column, and pelvis; diagnosed as multiple bone metastases of prostate cancer.

\section{Clinical Procedures and Outcomes}

At the time of the patient's first visit to his local dentist, he had already been on luteinizing hormone releasing hormone (LH-RH) therapy and monthly zoledronic acid for 7 months. Zoledronic acid was administered 7 times at doses of $28 \mathrm{mg}$. Three months later, he visited our department for extraction of the tooth which was giving him pain. No extraction was performed, however, due to concern that it might result in ONJ, and cleaning and medication with an antibacterial agent were selected instead.

Four months later, the patient was still undergoing chemotherapy and the pain in his tooth had become intolerable. Therefore, the mandibular molars were extracted with the agreement of the patient and urology 
Table 1 Treatment course

\begin{tabular}{|c|c|}
\hline Aug. XX00 & $\begin{array}{l}\text { First medical examination at Department of Urology. PSA 2,242 ng/ml. Palpation revealed } \\
\text { stone-like hardness. }\end{array}$ \\
\hline Sept. XX00 & $\begin{array}{l}\text { Biopsy: Gleason score } 5+4 \text {. Bone scintigraphy showed bone metastasis (Fig. 1). } \\
\text { [Diagnosis] Prostate cancer-prone bone metastasis. }\end{array}$ \\
\hline Nov. XX00 & Commencement of LH-RH hormonotherapy and zoledronic acid administration. \\
\hline Sept. XX01 & $\begin{array}{l}\text { First visit to our department with chief complaint comprising request for extraction of } \\
\text { mandibular left molar. } \\
\text { Was referred to us from local dental clinic. No extraction performed due to risk of ONJ. }\end{array}$ \\
\hline Jan. XX02 & $\begin{array}{l}\text { Torpidity of left nervus mentalis region appeared. Discharge of pus and exacerbation of pain in } \\
\text { mandibular left first molar. } \\
\text { Mandibular left first molar tooth extraction was performed, as chemotherapy could not be } \\
\text { continued due to pain in jawbone. } \\
\text { Subsequent epithelization of socket was good, and torpidity of left nervus mentalis disappeared. }\end{array}$ \\
\hline Aug. XX02 & ONJ developed around maxillary left premolar (Fig. 2 A, B). \\
\hline Feb. XX03 & ONJ expanded around maxillary left premolar (Fig. 2 C, D). \\
\hline Aug. XX03 & $\begin{array}{l}\text { Torpidity of left nervus mentalis region showed relapse; ONJ developed in mandibular left } \\
\text { molar region (Fig. } 2 \text { E-H). }\end{array}$ \\
\hline Jan. XX04 & $\begin{array}{l}\text { ONJ expanded further around maxillary left premolar; maxillary left canine became detached } \\
\text { (Fig. } 3 \mathrm{~A}-\mathrm{C} \text { ). }\end{array}$ \\
\hline Dec. XX04 & ONJ expanded in left maxilla and mandible (Fig. 3 D-G). \\
\hline Jan. XX05 & Left jaw position shifted. CT image showed pathological fracture of left mandible (Fig. 4 A, B). \\
\hline May. XX05 & Abscess and fistula formation developed in left sub-mandible (Fig. 4 C, D). \\
\hline Jun. XX05 & $\begin{array}{l}\text { Bleeding from mandibular ONJ region and impaired consciousness necessitated emergency } \\
\text { hospitalization. } \\
\text { At } 6 \text { days after hospitalization. Bleeding from mandibular ONJ region occurred } 3 \text { times within } \\
\text { short period; patient died of hemorrhagic shock. }\end{array}$ \\
\hline
\end{tabular}

department doctors. Zoledronic acid administration had reached a total dose of $48 \mathrm{mg}$ at this point, with the last dose being given 3 months earlier. An ampicillin drip was selected as the preoperative antibacterial agent. The surface of the bone of the extraction socket was dry. Re-epithelialization was good in the wound of the extracted teeth, and progress was considered to be good. After a while, chemotherapy was resumed.

Seven months later, the patient complained of sudden onset pain in the region of the left maxillary molars. A follow-up examination revealed that the alveolar bone of the maxilla was exposed, and mobility was evident in the surrounding teeth (Fig. 2). Treatment for this comprised cleaning and administration of antibiotics. Spontaneous tooth loss continued to occur, however, together with clear evidence of ONJ on a large scale. Therefore, in addition to administration of antibiotics and analgesics, occlusal adjustment was performed and necrotic tissue excised together with any remaining sharp-edged bone.

Recurrent pain from the ONJ of the maxilla worsened as the course of chemotherapy progressed, but slightly ameliorated during washout periods. At this point, tegafur-uracil, enzalutamide, flutamide, estramustine, cabazitaxel, and abiraterone acetic ester were being administered as adjunct therapies for the prostate cancer.

At 12 months after the onset of ONJ of the maxilla, torpor of the left mental nerve region was also evident, in addition to pain and bone exposure around the left mandibular molars (Fig. 3).

Cleaning was subsequently performed and antibiotics administered, but bone exposure continued to expand, accompanied by repeated infection. The ONJ gradually progressed and, at 17 months, the patient com- 

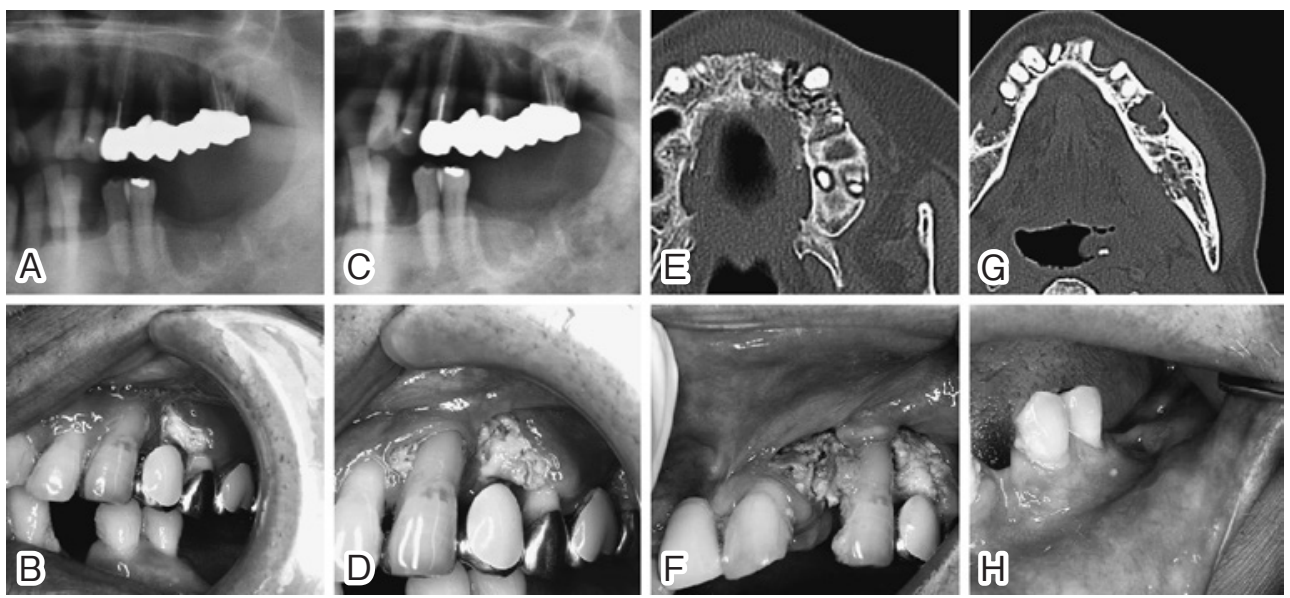

Fig. 2 Osteonecrosis of jaw (ONJ) images and oral cavity findings

A, C: Simple X-ray images; E, G: Computed tomography images; B, D, F: Progress in upper jaw ONJ; H: Mandibular ONJ

A, B: ONJ had developed around maxillary left premolar.

C, D: ONJ had expanded around maxillary left premolar.

E, F, G, and H: Torpidity of left nervus mentalis region showed relapse; ONJ had developed in mandibular left molar region.
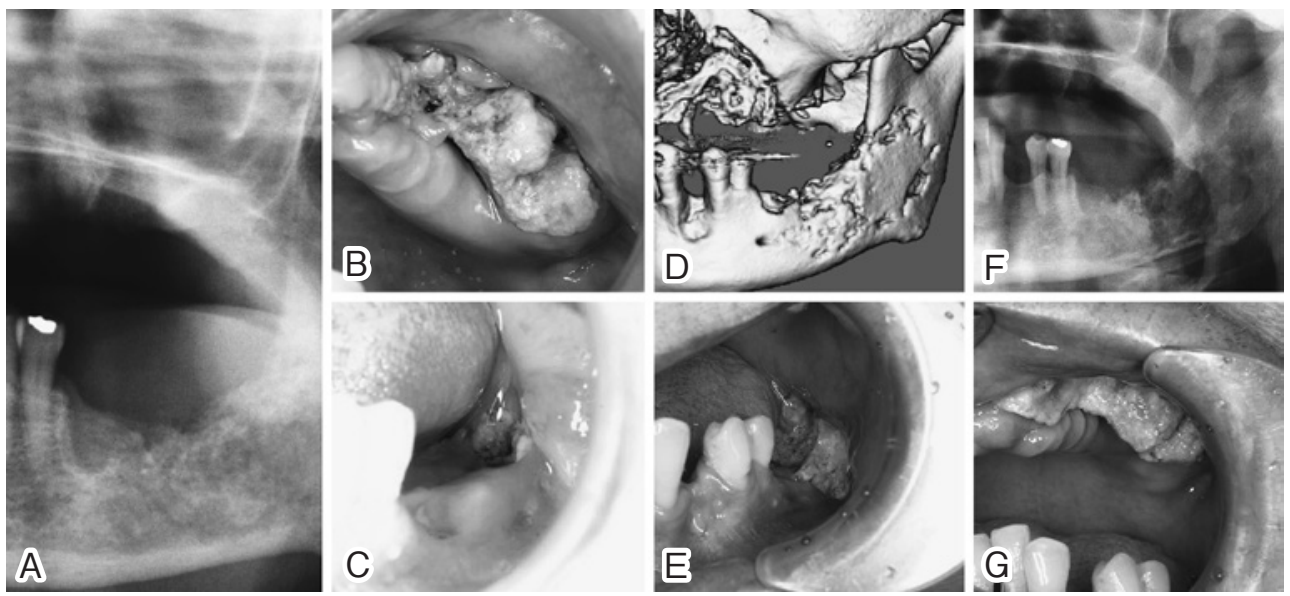

Fig. 3 Images of osteonecrosis of jaw (ONJ), enlargement, and oral cavity findings

A, B, C: ONJ showed more expansion around maxillary left premolar; maxillary left canine was detached. D, E, F and G: ONJ showed expansion in left maxilla and mandible.

plained of jaw deviation. Subsequent examination revealed a pathological fracture (Fig. 4).

At this point, 5 years had passed since the patient was first admitted. Pain control had become difficult, and mandible detachment and jaw position reconstruction were dis- cussed. Several days later, there was a hemorrhage from the portion of the jaw affected by ONJ, and the patient showed impaired consciousness. The patient was then admitted to hospital as an emergency case. At this time, radical treatment of the $\mathrm{ONJ}$ was considered necessary to control the bleeding, and a plan 

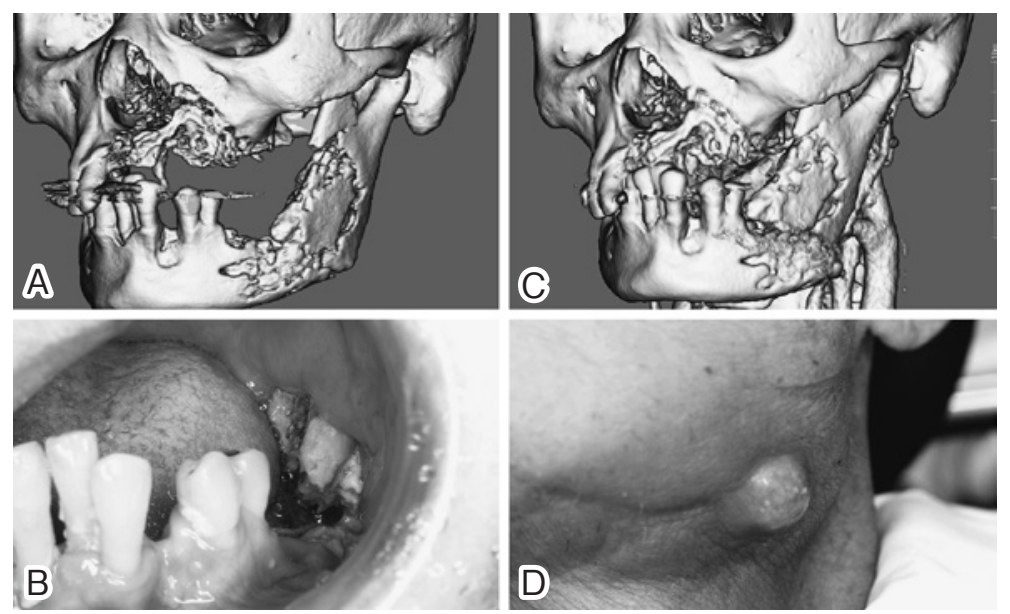

Fig. 4 Photographs of pathological fracture within and outside oral cavity

A, B: Left jaw position showed shift. CT image showed pathological fracture of left mandible.

C, D: Abscess and fistula formation at left submandibular.

was made for embolization based on the assumption that the patient's general condition would stabilize. The plan now was to detach and reconstruct the portion of the mandible affected to bring about a temporary arrest of hemorrhage. Early in the morning of the 6th day after hospitalization, hemorrhaging from the necrotic portion of left mandible occurred, and measures were taken to stop the bleeding with styptics and sutures. Nonetheless, after several hours, a large amount of bleeding involving the inferior alveolar artery occurred again from the same lesion and the patient fell into a state of shock. His family did not desire procedures to prolong life, and the patient died of hemorrhagic shock.

\section{Discussion}

Approximately 42,000 patients are diagnosed with prostate cancer in Japan each year. In 2020, it is expected that the yearly mortality rate will increase to 21,000 patients $^{7,20}$. With prostate cancer, there is an extremely high 10-year survival rate if the diagnosis is made early ${ }^{10}$. In advanced cases, such as those involving distant metastases, the standard strategy is the application of hormone treatment in conjunction with docetaxel combination therapy.

Hormone therapy is effective in at least $90 \%$ of such patients. Over half of highly advanced cases with distant metastases become insensitive to hormone therapy after several years, however. Therefore, it is predicted that the number of highly advanced cases in which anti-bone resorptive agents have to be used will increase, and this is especially likely to be associated with elderly patients whose general health status is already poor due to the rapid aging of the Japanese population.

In the AAOMS position paper ${ }^{25)}$, the risk of occurrence of $\mathrm{ONJ}$ was found to be between $0.7 \%$ and $6.7 \%$ in patients treated with zoledronic acid, as opposed to between $0 \%$ and $0.019 \%$ in placebo groups ${ }^{9,21,24,27)}$. Similarly, in a systematic review of randomized control studies $^{9,21,23,24)}$, the risk of ONJ occurrence was indicated to be $1 \%$ with treatment as opposed to between 50 and 100 times greater than that if left untreated. Additionally, outcomes in groups treated with denosumab, a RANKL monoclonal antibody drug, were comparable with those in patients treated with zoledronic 
$\operatorname{acid}^{24,26)}$

Concurrent use of bevacizumab, a multikinase inhibitor, with zoledronic acid increases risk of ONJ to $0.9 \%^{14,15,18,23)}$. Risk has also been reported to increase with concurrent use of sunitinib, a tyrosine kinase inhibitor, and zoledronic $\operatorname{acid}^{1-6,8,15,17,29)}$. These findings suggest the need to consider carefully the synergistic effects of concurrent chemotherapy in diagnosing MRONJ.

The AAOMS position paper ${ }^{25)}$ recommends symptomatic treatment, mouth rinse, and pain control as the main methods of management in treating cases of MRONJ up until stage 2 disease. It also recommends debridement only as a means of relieving soft tissue irritation and achieving infection control.

Furthermore, aggressive surgical procedures, such as surgical debridement or resection for longer-term palliation of infection and pain, is only recommended in cases of stage 3 disease $\mathrm{e}^{9,19,26,28,31}$.

Good results have been reported with aggressive surgical treatment such as reconstructive surgery following mandible detachment when no neovascularization was present $^{12,13,28)}$. Furthermore, some studies from $\operatorname{Japan}^{1,2,16,30)}$ have reported that it is possible to control ONJ by aggressive surgery or prompt reconstructive surgery in breast cancer patients on BP drugs. They also note that mandible detachment of the area affected or up to its borders may be necessary in some cancer patients, as long as the general health condition otherwise allows.

In determining whether to select aggressive surgical treatment, the oral surgeon must take into account a number of factors, including a poor prognosis in terms of life expectancy and its potential effect on the patient's QOL. This will be a difficult decision in patients already under palliative care.

In the present case, the patient had castration-resistant prostate cancer with a poor prognosis. The patient had survived for a long period of 6 years, however, owing to successful medication with hormone therapy and docetaxel. Moreover, this patient had stage-3 MRONJ and was undergoing long-term pallia- tive treatment for infection and pain. Therefore, mandible resection was considered. It was difficult to determine the optimal timing for surgical treatment, however, due to the worsening general health of the patient as a side effect of chemotherapy. Consultations were carried out with radiologists regarding embolization of the inferior alveolar artery in the mandible due to pathological fracture. Surgical intervention would have been appropriate earlier, perhaps at stage 2 , where we may have been able to halt the necrotic progress and subsequent fatal bleeding.

In the future, as the prevalence of cancer increases with the aging of the population, we believe that the prevalence of ONJ among the elderly will probably increase, and the scenario is likely to be further complicated by the development of various drugs, such as molecularly-targeted therapies. We believe that the present case could be taken as representative of the kind of problems that are likely to be encountered in elderly patients in poor general health and with stage-3 disease.

We believe that the oral surgeon will need to consider the feasibility of surgical treatment at stage 2 with ONJ, while the patient is still in good general health and capable of undergoing such treatment. An increase in the rate of surgical treatment of MRONJ at stage 2 would lead to a change in the therapeutic strategy for stage-3 disease.

\section{Conclusion}

Here, we have summarized our experience of a case involving hemorrhage due to osteonecrosis of the jaw resulting from ONJ caused by BP drugs for bone metastasis from prostate cancer.

\section{Acknowledgements}

We would like to express our gratitude to the doctors of the Urology Department of the Hachioji Hospital of Tokai University who provided special assistance and guidance. 


\section{Conflicts of Interest}

There are no conflicts of interest that need to be disclosed with regard to this paper.

\section{References}

1) Asaka T, Makino S, Takano M, Abe T, Abiko Y, Kitagawa Y (2013) Immediate reconstruction after segmental mandiblectomy in a patient with refractory osteomyelitis of the jaw cased by oral bisphosphonate therapy. Nihon Koku Geka Gakkai Zasshi 59:182-186. (in Japanese)

2) Asaka Y, Makita H, Yoneyama K, Yamashita T, Kato K, Shibata T (2009) Successful surgical treatment of a case of bisphosphonate-related osteonecrosis of the mandible. Nihon Koku Geka Gakkai Zasshi 55:354-358. (in Japanese)

3) Ayllon J, Launay-Vacher V, Medioni J, Cros C, Spano JP, Oudard S (2009) Osteonecrosis of the jaw under bisphosphonate and antiangiogenic therapies: Cumulative toxicity profile? Ann Oncol 20:600-601.

4) Balmor GR, Yarom N, Weitzen R (2012) Druginduced palate osteonecrosis following nasal surgery. Isr Med Assoc J 14:193-194.

5) Beuselinck B, Wolter P, Karadimou A, Elaidi R, Dumez H, Rogiers A, Van Cann T, Willems L, Body JJ, Berkers J, Van Poppel H, Lerut E, Debruyne P, Paridaens R, Schöffski P (2012) Concomitant oral tyrosine kinase inhibitors and bisphosphonates in advanced renal cell carcinoma with bone metastases. Br J Cancer 107:1665-1671.

6) Bozas G, Roy A, Ramasamy V, Maraveyas A (2010) Osteonecrosis of the jaw after a single bisphosphonate infusion in a patient with metastatic renal cancer treated with sunitinib. Onkologie 33:321-323.

7) Cancer statistics (2003) Death rates adjusted for age due to malignant neoplasms (19502001); http://www.ncc.go.jp/jp

8) Christodoulou C, Pervena A, Klouvas G, Galani E, Falagas ME, Tsakalos G, Visvikis A, NikolakopoulouA, Acholos V, Karapanagiotidis G, Batziou E, Skarlos DV (2009) Combination of bisphosphonates and antiangiogenic factors induces osteonecrosis of the jaw more frequently than bisphosphonates alone. Oncology 76:209-211.

9) Coleman R, Woodward E, Brown J, Cameron D, Bell R, Dodwell D, Keane M, Gil M, Davies C, Burkinshaw R, Houston SJ, Grieve RJ, Barrett-Lee PJ, Thorpe H (2011) Safety of zoledronic acid and incidence of osteonecro- sis of the jaw (ONJ) during adjuvant therapy in a randomised phase III trial (AZURE: BIG 01-04) for women with stage II/III breast cancer. Breast Cancer Res Treat 127:429-438.

10) Critz FA, Benton JB, Shrake P, Merlin ML (2013) 25-Year disease-free survival rate after irradiation for prostate cancer calculated with the prostate specific antigen definition of recurrence used for radical prostatectomy. J Urol 189:878-883.

11) Cummings SR, San Martin J, McClung MR, Siris ES, Eastell R, Reid IR, Delmas P, Zoog HB, Austin M, Wang A, Kutilek S, Adami S, Zanchetta J, Libanati C, Siddhanti S, Christiansen C; FREEDOM Trial (2009) Denosumab for prevention of fractures in postmenopausal women with osteoporosis. N Engl J Med 361:756-765.

12) Engroff SL, Kim DD (2007) Treating bisphosphonate osteonecrosis of the jaws: Is there a role for resection and vascularized reconstruction. J Oral Maxillofac Surg 65:2374-2385.

13) Ferrari S, Bianchi B, Savi A, Poli T, Multinu A, Balestreri A, Ferri A (2008) Fibula free flap with endosseous implants for reconstructing a resected mandible in bisphosphonate osteonecrosis. J Oral Maxillofac Surg 66:999-1003.

14) Fleissig Y, Regev E, Lehman H (2012) Sunitinib related osteonecrosis of jaw: A case report. Oral Surg Oral Med Oral Pathol Oral Radiol 113:e1-e3.

15) Guarneri V, Miles D, Robert N, Diéras V, Glaspy J, Smith I, Thomssen C, Biganzoli L, Taran T, Conte P (2010) Bevacizumab and osteonecrosis of the jaw: Incidence and association with bisphosphonate therapy in three large prospective trials in advanced breast cancer. Breast Cancer Res Treat 122:181-188.

16) Hariya Y, Sekiguchi T, Okita M, Harada M, Ohuchi T (2008) Surgical intervention of osteonecrosis of the maxilla associated with bisphosphonate therapy: report of a case. Nihon Koku Geka Gakkai Zasshi 54:413-417. (in Japanese)

17) Hoefert S, Eufinger H (2010) Sunitinib may raise the risk of bisphosphonate-related osteonecrosis of the jaw: presentation of three cases. Oral Surg Oral Med Oral Pathol Oral Radiol Endod 110:463-469.

18) Koch FP, Walter C, Hansen T, Jäger E, Wagner $\mathrm{W}$ (2011) Osteonecrosis of the jaw related to sunitinib. J Oral Maxillofac Surg 15:63-66.

19) Kumar SK, Gorur A, Schaudinn C, Shuler CF, Costerton JW, Sedghizadeh PP (2010) The role of microbial biofilms in osteonecrosis of the jaw associated with bisphosphonate therapy. Curr Osteoporos Rep 8:40-48.

20) Kuroishi T, Hirose K (2004) Prediction of the 
cancer death rate in Japan. Cancer statistics white paper-cancer-bearing/death/prognoses 2004, Oshima A, Kuroishi T, Tajima K (Eds),pp.219-234,Shinoharashinsha Publishers, Tokyo.

21) Mauri D, Valachis A, Polyzos IP, Polyzos NP, Kamposioras K, Pesce LL (2009) Osteonecrosis of the jaw and use of bisphosphonates in adjuvant breast cancer treatment: A meta-analysis. Breast Cancer Res Treat 116:433-439.

22) Nicolatou-Galitis O, Migkou M, Psyrri A, Bamias A, Pectasides D, Economopoulos T, Raber-DurlacherJE, Dimitriadis G, Dimopoulos MA (2012) Gingival bleeding and jaw bone necrosis in patients with metastatic renal cell carcinoma receiving sunitinib: report of 2 cases with clinical implications. Oral Surg Oral Med Oral Pathol Oral Radiol 113:234-238.

23) Papapoulos S, Chapurlat R, Libanati C, Brandi ML, Brown JP, Czerwiński E, Krieg MA, Man Z, Mellström D, Radominski SC, Reginster JY, Resch H, Román Ivorra JA, Roux C, Vittinghoff E, Austin M, Daizadeh N, Bradley MN, Grauer A, Cummings SR, Bone HG (2012) Five years of denosumab exposure in women with postmenopausal osteoporosis: results from the first two years of the FREEDOM extension. J Bone Miner Res 27:694-701.

24) Qi WX, Tang LN, He AN, Yao Y, Shen Z (2014) Risk of osteonecrosis of the jaw in cancer patients receiving denosumab: a meta-analysis of seven randomized controlled trials. Int J Clin Oncol 19:403-410.

25) Ruggiero SL, Dodson TB, Fantasia J, Goodday R, Aghaloo T, Mehrotra B, O'Ryan F; American Association of Oral and Maxillofacial Surgeons (2014) American Association of Oral and Maxillofacial Surgeons position paper on medication-related osteonecrosis of the jaw2014 update. J Oral Maxillofac Surg 72:19381956.

26) Scagliotti GV, Hirsh V, Siena S, Henry DH, Woll PJ, Manegold C, Solal-Celigny P, Rodriguez G, Krzakowski M, Mehta ND, Lipton L, García-SáenzJA, Pereira JR, Prabhash $\mathrm{K}$, Ciuleanu TE, Kanarev V, Wang $\mathrm{H}$, Balakumaran A, Jacobs I (2012) Overall survival improvement in patients with lung cancer and bone metastases treated with denosumab versus zoledronic acid: Subgroup analysis from a randomized phase 3 study.J Thorac
Oncol 7:1823-1829.

27) Sedghizadeh PP, Kumar SK, Gorur A, Schaudinn C, Shuler CF, Costerton JW (2008) Identification of microbial biofilms in osteonecrosis of the jaws secondary to bisphosphonate therapy. J Oral Maxillofac Surg 66: $767-775$.

28) Seth R, Futran ND, Alam DS, Knott PD (2010) Outcomes of vascularized bone graft reconstruction of the mandible in bisphosphonaterelated osteonecrosis of the jaws. Laryngoscope 120:2165-2171.

29) Smidt-Hansen T, Folkmar TB, Fode K, Agerbaek M, Donskov F (2013) Combination of zoledronic acid and targeted therapy is active but may induce osteonecrosis of the jaw in patients with metastatic renal cell carcinoma. J Oral Maxillofac Surg 71:1532-1540.

30) Suzuki T, Hamada U, Takahashi M, Karakida K, Sakamoto H (2017) A clinical research on prognoses of the medication-related osteonecrosis of the jaw (MRONJ). Jpn J Oral Diag/ Oral Med 30:327-332.

31) Wanger G, Gorby Y, El-Naggar MY, Yuzvinsky TD, Schaudinn C, Gorur A, Sedghizadeh PP (2013) Electrically conductive bacterial nanowires in bisphosphonate-related osteonecrosis of the jaw biofilms. Oral Surg Oral Med Oral Pathol Oral Radiol 115:71-78.

32) Yoneda T, Hagino $H$, Sugimoto $T$, Ohta $H$, Takahashi S, Soen S, Taguchi A, Toyosawa S, Nagata T, Urade M (2010) Bisphosphonaterelated osteonecrosis of the jaw: position paper from the Allied Task Force Committee of Japanese Society for Bone and Mineral Research, Japan Osteoporosis Society, Japanese Society of Periodontology, Japanese Society for Oral and Maxillofacial Radiology, and Japanese Society of Oral and Maxillofacial Surgeons. J Bone Miner Metab 28:365-383.

\section{Correspondence:}

Dr. Taiki Suzuki

Department of Oral and Maxillofacial

Surgery,

Hachioji Hospital Tokai University,

1838 Ishikawa-cho, Hachioji,

Tokyo 192-0032, Japan

E-mail: suzukitaiki@tdc.ac.jp 